\title{
Doença do neurônio motor dos eqüinos: relato dos primeiros casos na América do Sul
}

CORRESPONDENCE TO:

Márcio Rubens Graf Kuchembuck Departamento de Clínica

Veterinária

Faculdade de Medicina Veterinária

e Zootecnia da UNESP

18618-000 - Botucatu - SP - Brasil

\section{Equine motor neuron disease: report on the first cases in South America} Rogério Martins AMORIM; Márcio Rubens Graf KUCHEMBUCK; Roberto Calderon GONÇALVES ; Renée I.AUFER;
Júlio Lopes SEQUEIRA; Vitalino Dal PAI ; Raimundo Souza LOPES

\section{RESUMO}

\begin{abstract}
Foram realizados estudos dos casos de 6 eqüinos de raças e idades diferentes, apresentando um quadro clínico caracterizado principalmente por emagrecimento progressivo, atrofia e fraqueza muscular generalizada, fasciculações e tremores musculares, alternância constante de apoio dos membros posteriores, deslocamento do apoio dos membros anteriores caudalmente e dos membros posteriores cranialmente, decúbito lateral prolongado e morte. A confirmação do diagnóstico ocorreu mediante exames histopatológicos da medula espinhal, observando-se uma degeneração e perda dos neurônios localizados no corno ventral do $\mathrm{H}$ medular, sendo a lesão mais grave na região das intumescências cervical e lombar. As biópsias musculares revelaram alterações neuromusculares caracterizadas por atrofia neurogênica, complementando o diagnóstico de doença do neurônio motor. A atrofia e fraqueza muscular progressiva, assim como a degeneração dos neurônios motores, são similares àquelas descritas em humanos com esclerose lateral amiotrófica esporádica ou doença de Lou Gehrig's.
\end{abstract}

UNITERMOS: Neurônios; Equidae; Atrofia muscular; Desnervação.

\section{INTRODUÇÄO}

$\longrightarrow$ ummings et al. relataram nos Estados Unidos uma neuronopatia, a qual denominaram de doença do neurônio motor dos equiinos (Equine Motor Neuron Disease - EMND). Após um estudo retrospectivo realizado no período de janeiro de 1985 a setembro de 1991, em que 32 eqüinos portadores do processo foram identificados por Mohammed et al." (1993), este número subiu para 45 casos, todos na América do Norte". Recentemente, foram reconhecidos nos Estados Unidos 67 casos conlirmados e alguns outros suspeitos na lnglaterra ${ }^{a 2}$.

A doença do neurônio motor é caracterizada patologicamente por uma degeneração dos neurônios localizados na medula espinhal e de alguns núcleos motores do tronco cerebral dos equíideos ${ }^{2+1123}$, manifestando-se clinicamente por atrolia muscular progressiva e fraqueza intensa, similares àquelas descritas na espécie humana, em indivíduos portadores de esclerose lateral amiotrófica ou doençá de Lou Gehrig's

A doença do neurônio motor dos eqüinos e a esclerose lateral amiotrófica no homem apresentam semelhanças clínicas e anatomopatológicas, não possuindo entretanto etiologia esclarecida ${ }^{+18,24}$. Nos últimos dois anos e meio, sinais clínicos sugestivos desta enfermidade vêm sendo observados no Regimento de Cavalaria 9 de Julho da Polícia Militar de São Paulo, acometendo 20 eqüinos, sendo que 6 foram encaminhados ao Serviço de Clínica de Grandes Animais da Faculdade de Medicina Veterinária e Zootecnia da UNESP em Botucatu, objetivando o presente relato.

\section{MATERIAL, E MÉTODO}

O material utilizado consistiu em 6 animais apresentando atrofia e fraqueza muscular, enviados à Clínica de Grandes Animais da FMVZ da UNESP em Botucatu (Quad. 1), o. quais foram submetidos a exame clínico geral e neurológico. Os exames complementares constituíram-se da determinação da aspartato-aminotransferase (AST) e creatinoquinase (CK) séricas pelo "kil" comercial CELM e proteína liquórica pelo método do ácido tricloroacético ${ }^{17}$. Dos 6 eqüinos, somente 02 tiveram morte espontânea (casos 1 e 2), sendo os demais submetidos à eutanásia (casos 3, 4, 5 e 6). Realizaram-se exames necroscópicos, de acordo com técnicas de rotina, sendo colhi- 


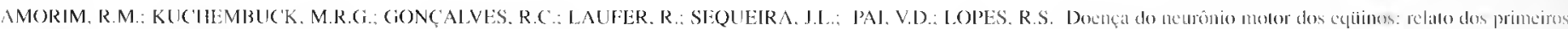

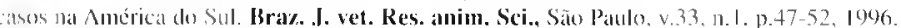

dos para histopatologia: encéfalo, medula espinhal, músculos, nervos periféricos, fígado e rins. Este material foi fixado em solução tamponada de formol a $10 \%$, processado conforme protocolo do Serviço de Anatomia Patológica da FMVZ da UNESP em Botucatu, corando-se os cortes, que variavam de 5 a 7 $4 \mathrm{~m}$. pelos métodos de hematoxilina-eosina e Woelcke para bainha de mielinat ${ }^{14}$. Foram colhidos segmentos de medula espinhal de tum animal controle para efeito comparativo (Figs. $5 \mathrm{~A}$ e $5 \mathrm{C}$ ).

Fragmentos dos músculos braquiocefálico, glúteo profundo c semimembranoso foram colhidos dos casos 3, 4 e 6; sendo imersos em n-hexana, a $-70^{\circ} \mathrm{C}$ previamente resfriados em nitrogênio líquido e a seguir transferidos para um micrótomo criostato a $-20{ }^{\circ} \mathrm{C}$. Várias séries de cortes histológicos com

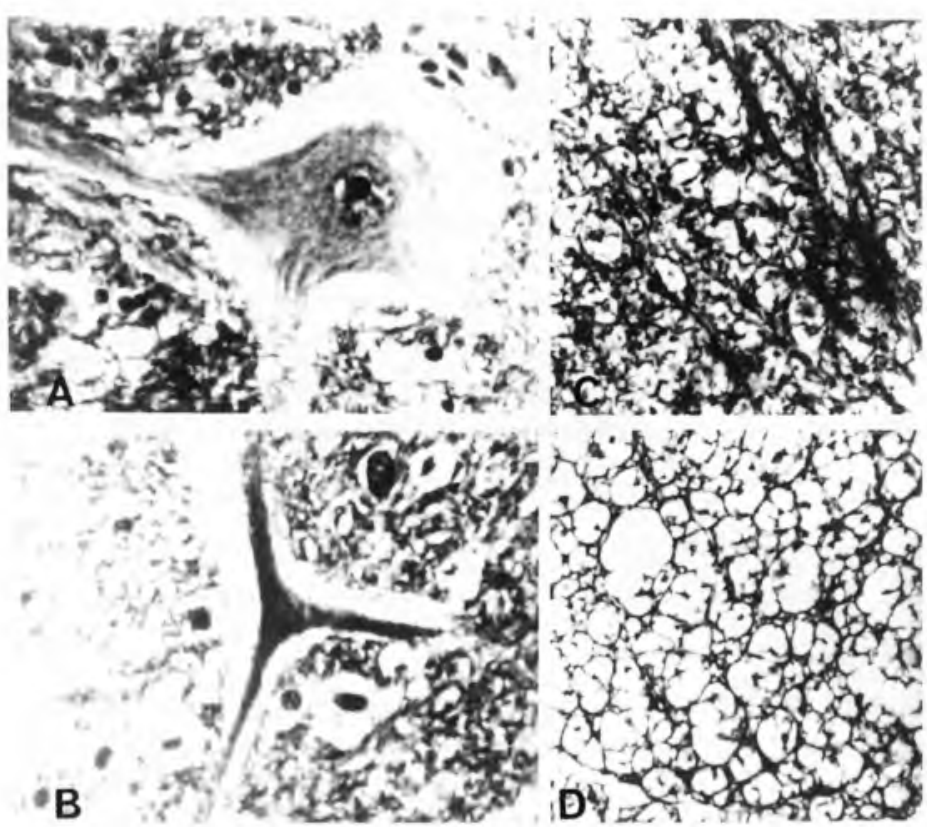

Figura 5

A: Neurônio motor inferior. Animal controle. Coloração de Woelcke $4000 x$

B: Caso 3. Neurônio motor inferior em degeneração. Coloração de Woelcke 4000x

C: Substância branca medular. Animal controle. Coloração de Woelcke 2500x.

D: Caso 3. Substância branca medular, Desmielinização. Coloração de Woelcke.

8 fum de espessura foram corados de acordo com os métodos de hematoxilina, tricrômico de Mallory, pelas técnicas do ácido periódico-Schiff (PAS), nicotinamida adenina dinucleotídio tetrazólio redutase (NADH-TR), ATPase miofibrilar (m-ATPase) em pH 9.4, após pré-incubação em meio ácido (pH 4.35) e em meio alcalino (pH 10.40), succinato desidrogenase (SDH), esterase inespecífica (EI) e oil red ${ }^{*}$. Com base na área, na intensidade e distribuição citológica dos produtos finais das reações histoquímicas, as fibras musculares foram classificadas em SO (Slow Oxydative), FOG (Fast Oxydative Glycolitic) e FG (Fast Glycolitic) ".

\section{RESULTADOS}

\section{Sinais clínicos}

A manifestação dos sinais clínicos foi variável conforme o estágio de evolução da doença. A fase incicial caracterizou-se por fasciculações musculares leves, mais evidentes na regiāo da escápula e membros posteriores, que se tornavam exacerbadas após exercícios (principalmente em rampas), evoluindo para tremores musculares. Alguns animais apresentavam hiperestesia ao toque, principalmente da musculatura cervical e escapular. A freqüente alternância de apoio dos membros posteriores, bem como o seu deslocamento cranial e dos membros anteriores caudalmente, foi observada em todos os animais acometidos (Fig. I A, I B, IC). Os sinais desenvolveramse gradativamente, de um a vários meses, com emagrecimento progressivo, apesar de o apetite variar de normal a voraz em alguns casos. Evidenciou-se, assim, um processo de atrofia muscular generalizada e conseqüente fraqueza, caracterizada no exame clínico neurológico pelo arrastar das pinças dos membros posteriores, hipometria, incapacidade de erguer a cabeça, sudorese excessiva após exercício mínimo e episódios intermitentes de decúbito esternal e lateral. A atrofia foi mais acentuada nos grupos musculares da região lombar, membros posteriores (quadríceps, semimembranoso e semitendinoso). na região do pescoço (braquiocelálico e esternocefálico) e da escápula. Os equiinos frequientemente adotavam atitude de decúbito esternal e lateral por longos períodos, aumentando progressivamente a dificuldade do animal em manter-se em estação, permanecendo em decúbito lateral até a morte espontânea ou eutanásia. Nenhum dos 06 animais apresentava envolvimento encefálico, uma vez que possuíam estatdo mental normal e ausência de alterações dos nervos cranianos. Deficiências proprioceptivas não foram observadas durante o exame neurológico. (Os sinais clínicos estão resumidos na Tab. 1 .

\section{Patologia clínica}

Os achados laboratoriais estão restritos a eventuais alterações da AST e CK séricas e proteína liquórica, relacionados na Tab. 2.

\section{Biópsias musculares}

Conforme revelado pelas técnicas morfológicas e histoquímicas empregadas, os músculos estudados mostraram diferentes graus de alterações, sendo mais severas no músculo braculocelálico. Foram observadas fibras hipertróficas e arredondadas; outras angulosas e atróficas, sob a forma de grupos ou dispersas (Fig. 2A). As reações NADH-TR e SDH 

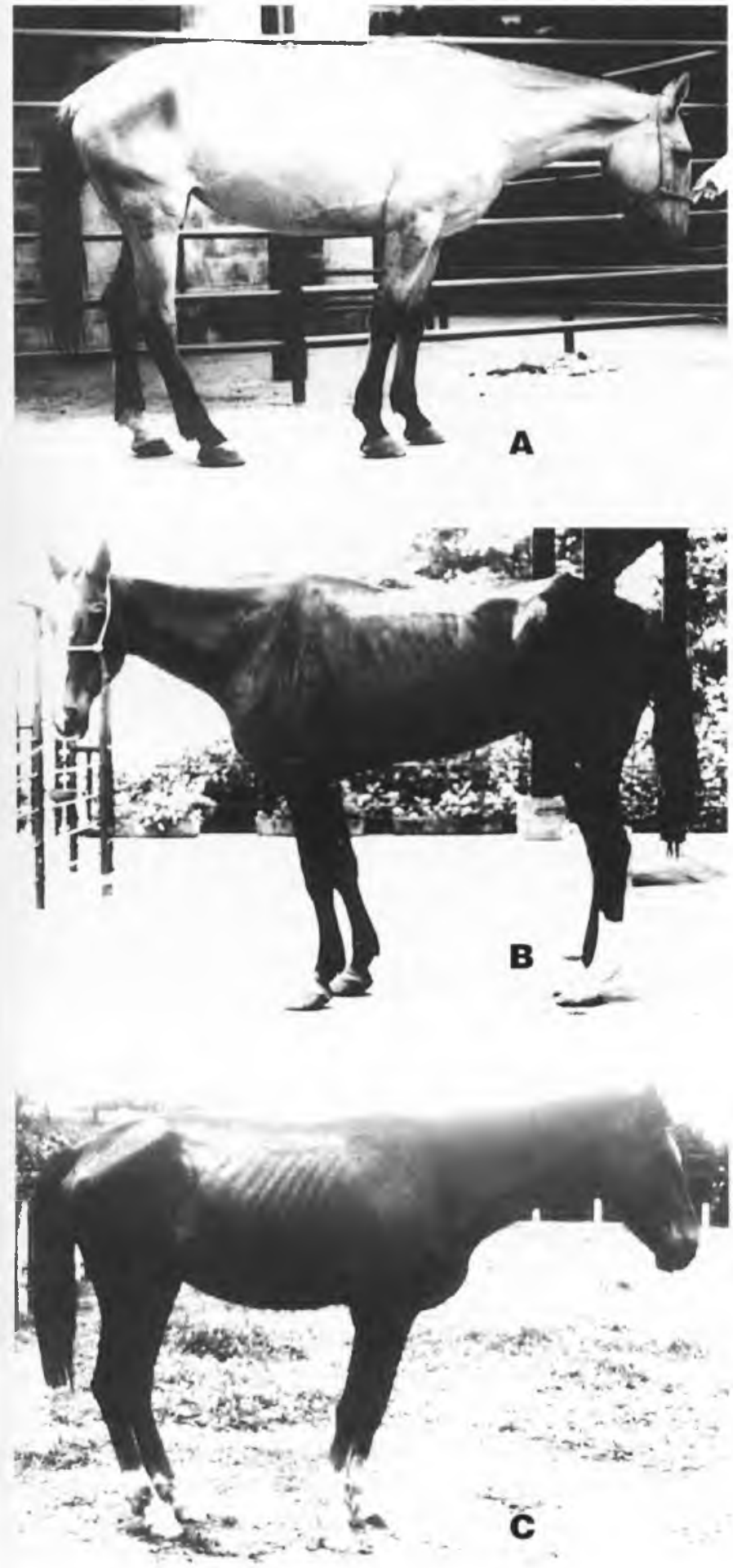

Figura 1

1A: Caso 3. Note o deslocamento do apoio dos membros posteriores cranialmente e dos anteriores caudalmente, atrofia muscular na regiāo cervical e lombar, dificuldade em manter a cabeça erguida.

1B: Atrofia muscular generalizada.

1C: Atrofia muscular e deslocamento do apoio dos membros. revelaram-se intensas nas fibras angulosas e alróficals. Nas fibras hipertróficas, o produto destas reações mostrou distribuição heterogênea, con áreas desprovidas de reação e ou tras sob a forma de agregados. A reatividade intensa nas fïras angulosass e atróficas caracterizou desenervação, encjuanto a distribuição anormal do produlo das reações para metabolismo aeróbico revelou lesão metabólica (Fig. 2B). A altrofia revelouse mais acentuada nas fïbras anaeróbicas de contração rápida FG (tipo 2B) e nas fïbras aeróbicas de contração rápicla Fo() (lipo 2A). A atrofia das fibras SO (tipo 1) mostrou-se menos intensa (Fig. 2C). A reação PAS revelou depleção total de glicogênio nas fibras atróficas. Já nas fïbras restantes a depleção mostrou-se parcial e de modo helerogeneo (Fig. 2D).

\section{Achados de necrópsia}

A necrópsia, observou-se em todos os animais intensa atrofia muscular acompanhada de edema e áreas focais claras na musculatura, estado nutricional ruim, congestão hepálica c dilatação do ventrículo direito. Os demais órgãos apresen-

Tabela 1

Manifestação dos sinais clínicos e evolução de seis animais com doença do neurônio motor dos eqüinos. Botucatu, 1993.

\begin{tabular}{|c|c|c|c|c|c|c|}
\hline \multirow[b]{2}{*}{ SINAIS CLÍNICOS } & \multicolumn{6}{|c|}{ CASOS } \\
\hline & 1 & 2 & 3 & 4 & 5 & 6 \\
\hline $\begin{array}{l}\text { Emagrecimento } \\
\text { progressivo }\end{array}$ & + & + & + & + & + & + \\
\hline Fasciculações & + & + & + & + & $+/-$ & + \\
\hline $\begin{array}{l}\text { Tremores } \\
\text { musculares }\end{array}$ & + & + & + & + & $+1-$ & + \\
\hline Hiperestesia & + & $+/-$ & + & + & - & + \\
\hline Apetite voraz & + & + & + & + & $+/-$ & + \\
\hline $\begin{array}{l}\text { Atrofia } \\
\text { muscular }\end{array}$ & + & + & + & + & + & + \\
\hline Fraqueza & + & + & + & + & + & + \\
\hline Hipometria & + & + & + & + & + & + \\
\hline $\begin{array}{l}\text { Troca constante do } \\
\text { apoio dos membros } \\
\text { posteriores }\end{array}$ & + & + & + & + & $+1-$ & + \\
\hline $\begin{array}{l}\text { Deslocamento } \\
\text { dos membros } \\
\text { posteriores } \\
\text { cranialmente e } \\
\text { dos anteriores } \\
\text { caudalmente }\end{array}$ & + & + & + & + & + & + \\
\hline $\begin{array}{l}\text { Sudorese } \\
\text { excessiva }\end{array}$ & $+1-$ & + & $+/-$ & + & - & + \\
\hline $\begin{array}{l}\text { Inabilidade em } \\
\text { erguer a } \\
\text { cabeça }\end{array}$ & + & + & + & + & + & - \\
\hline Decúbito & + & + & + & + & + & + \\
\hline Evolução & 1 mès & 4 meses & 2 meses & 50 dias & 2 meses & 1 mês \\
\hline
\end{tabular}




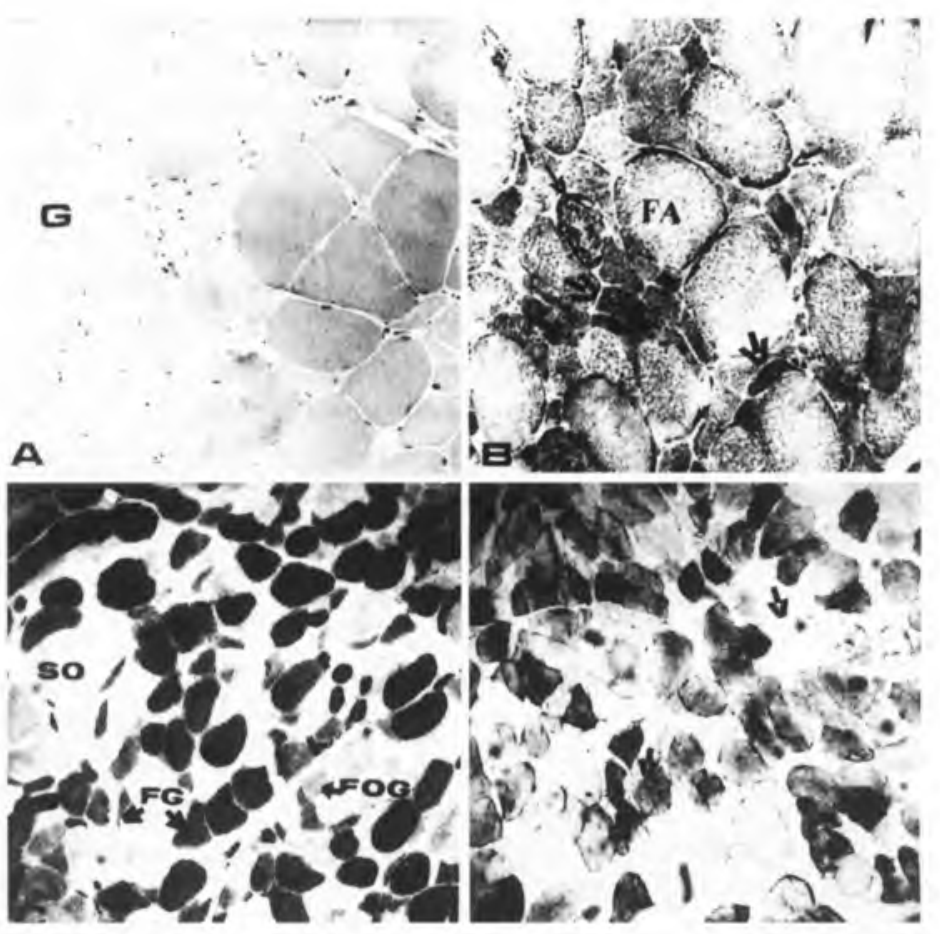

Figura 2

Músculo braquiocefálico. A: Fibras hipertróficas e arredondadas, fibras angulosas e atróficas em grupos $(G)$ e isoladas HE.200x. B: Fibras angulosas e atróficas com intensa reação NADH-TR (setas) e fibras hipertróficas aeróbicas (FA), com distribuição irregular do produto da reação (seta menor) NADH-TR.200x. C: Variável grau de atrofia das fibras FG e FOG, hipertrofia das fibras SO, m-ATPase, $\mathrm{pH} 10.4,80 \mathrm{x}$. D Fibras atróficas e hipertróficas com depleçāo total ou parcial do glicogênio (setas) PAS.200x.

tavam-se sem alterações macroscópicas significativas.

\section{Histopatologia}

() exame microscópico revelou diminuição marcante do número de corpos celulares dos neurônios motores do $\mathrm{H}$ medular. sendo mais severa na região ventral das intumescências cervical e lombar da medula espinhal (Fig. 3). Este quadro era representado por vacuolização da substância cinzenta medular, com lïguras de cicaltriz glial (ausência de soma neuronal) (Fig. 4), alguns neurônios irregulares, pálidos, sem núcleo visível (Fig. 5B), sofrendo neuronofagia e gliose vatriando de intensidade. A presença de neurônios tumelatos e cromatolíticos foi discreta, podendo-se observar em alguns deles corpúsculos ciroplasmáticos eosinolílicos, ovais ou esféricos. Em 3 casos (casos 2, 3 e 5) a gliose foi menos intensa e nolou-se um número ainda menor de corpos celulares. Os vatsos da substância brancat e cinzenta da medula e encéfalo apresentavam-se hiperêmicos. Nos cortes corados pelo método de Woelcke para bainha de mielina, foram observados desmiclinização dos axônios, representada pelo acentuado rendilhamento da substância branca (Fig. 5D); fragmentos de axônio sofrendo degeneração walleriana, com debris de mielina e macrófagos presentes nestas regiões. No lígado e rins de todos os animais observava-se intensal congestão passiva.

\section{DISCUSSÃO E CONCLUSÃO}

A determinação dos diagnósticos dilerenciais da doença do neurônio motor dos eqüinos inclui alecções que apresentam sinais clínicos de lesão de medula espinhal e ou sistema muscular. Dentre elas a micloencefalopatial degenerativa, a mieloencelalite prolozória, a encefalomielite e as mieloencefalopatias virais (raiva e herpesvírus eqüino) lambém podem acometer os neurônios motores da medula espinhal. Entretanto. o envolvimento destes neurônios na doenç̧a do neurônio motor difere tanto clínica como anatomopaltologicamente daquele encontrado nestas doenças's. Clinicamente. at maioria das afecções medulares em equiinos manifesta-se com déficits proprioceptivos". Na doença do neurônio motor dos equiinos, as deficiências proprioceptivas estão ausentes, diterenciando-a destas outras afecções medulares. A mieloencefalitè

Quadro 1

Identificação dos animais com Doença do Neurônio Motor dos Eqüinos. Botucatu, 1993.

\begin{tabular}{lcccc}
\hline Casos & Raça & $\begin{array}{c}\text { Sexo } \\
\text { (anos) }\end{array}$ & Idade & Origem \\
\hline 1 & $\begin{array}{c}\text { Mestiço PSI } \\
\text { Brasileiro }\end{array}$ & $\begin{array}{c}\text { Macho } \\
\text { Macho }\end{array}$ & $\begin{array}{c}10 \\
\text { de Hipismo }\end{array}$ & $\begin{array}{c}\text { São Paulo - SP } \\
\text { São Paulo - SP }\end{array}$ \\
& Mestiço PSI & Fêmea & 9 & São Paulo - SP \\
3 & Mestiço PSI & Macho & 17 & São Paulo - SP \\
4 & Mestiço PSI & Macho & 11 & Itapetininga - SP \\
5 & $\begin{array}{c}\text { Srasileiro } \\
\text { de Hipismo }\end{array}$ & Macho & 9 & São Paulo - SP \\
6 & & & \\
\hline
\end{tabular}

Fonte: Regimento de Cavalaria 9 de julho da Policia Militar de São Paulo.

protozoária pode resultar em degeneração focal ou multifocal de neurônios motores e degeneração da substância branca do encéfalo e medula espinhal, porém a fracueza resultante dos neurônios motores inferiores é localizada e freqüentemente assimétrica A polioencefalomielite calusada pelo vírus da raiva pode inicialmente produzir sinais clínicos sugestivos de doença do neurônio motor ". Entretanto a lesão rapidamente torna-se difusa sendo acompanhada por outros sinatis neurológicos ${ }^{516}$. A ausência clínica de disfunções encefálicàs nos casos estudados foi um fator imporante na exclusão dos problemas neurológicos que frequientemente se manilestam com encefalopatia.

No exame microscópico dessas afecções podem ser notados manguitos perivasculares; infiltrado inflamatório mono ou polimorfonuclear, corpúsculos de inclusão; ou até mesmo o protozoário presente no tecido nervoso. Além disso. as alterações 
AMORIM. R.M.; KUCHEMBUCK. M.R.G.; GONÇALVES. R.C.: LAUFER, R; SEQUEIRA. J.L.: PAI. V.D.: LOPES. R.S. Doença do neurônio molor dos eqüinos: relato dos primeiros casos na América do Sul. Braz. J. vet. Res. anim. Sei., São Paulo, v.33, n. I, p.47-52. 1996.

Tabela 2

Achados laboratoriais de seis animais com Doença do Neurônio Motor dos Eqüinos. Botucatu, 1993.

\begin{tabular}{|c|c|c|c|c|c|c|c|c|c|}
\hline & \multirow{2}{*}{$\begin{array}{l}\text { Valores } \\
\text { normais }\end{array}$} & \multicolumn{6}{|c|}{ Casos } & \multirow[b]{2}{*}{$x$} & \multirow[b]{2}{*}{$\mathrm{Sd}$} \\
\hline & & 1 & 2 & 3 & 4 & 5 & 6 & & \\
\hline $\mathrm{AST}_{\text {sérica }}$ & $58-94 \cup / L^{(a)}$ & 361,5 & 298,8 & 180,7 & 154,2 & 108,5 & 287,4 & 231,8 & 98,22 \\
\hline $\mathrm{CK}_{\text {sérica }}$ & $2,4-23,4 \mathrm{U} / \mathrm{L}^{(b)}$ & 98,4 & 32,8 & 34,4 & 66,4 & 65,4 & 106,6 & 67,33 & 30,95 \\
\hline $\begin{array}{l}\text { Proteína } \\
\text { liquórica }\end{array}$ & $5-100 \mathrm{mg} / \mathrm{d}^{(\mathrm{c})}$ & 40,0 & - & 142,5 & 144,0 & 49,9 & 58,8 & 87,04 & 51,74 \\
\hline
\end{tabular}

(a) PEARSON; GRAIG ${ }^{20}, 1980$

(b) KANEKO ${ }^{12}, 1989$

(c) MAYHEW ${ }^{17}, 1977$

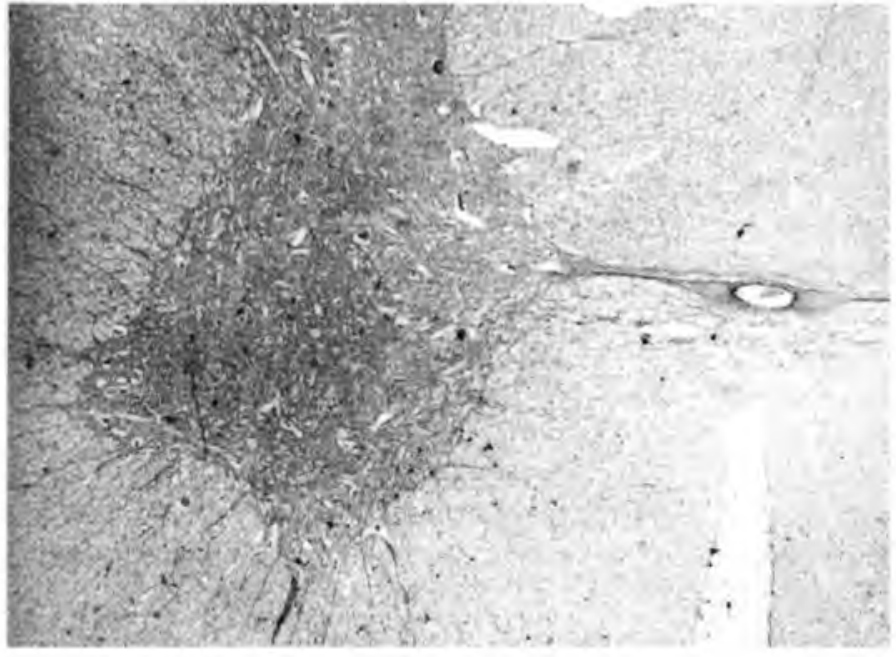

Figura 3

Caso 1. Corno ventral da medula espinhal. Porção lombar. Diminuição marcante dos neurônios motores inferiores. HE.200x

encefálicals são mais graves do que aquelas observadas na doença do neurônio motor, onde há, basicamente, gliose moderada.

A hiperproteinorraquia foi mais evidente em alguns animais que apresentavam progressão dos sinais clínicos. Esta elevação da proténa licjuórica pode refletir o processo de degeneração e morte dos neurônios da medula espinhal ". Nos animais cronicamente afetados espera-se que esta assuma valores normais para a espécie $\left(5-10(0 \mathrm{mg} / \mathrm{dl}){ }^{1+}\right.$. As médias dos valores de AST e CK, nos casos estudados (Tab. 2), estavam acima dos valores normais segundo Pearson; Graig ". 1980 e Kaneko '?. 1989, respectivamente. A altividade sérica das enzimas musculares (AST e CK) pode estar moderada ou acentuadamente clevada, na dependência de tremores intensos c ou decúbito frequiente. Porém, nos animais cronicamente atetatdos, estes valores tendem a retornar aos níveis normais ".

As biópsias musculares revelaram atrofia de fibrals dispersas e em grupos de ambos os tipos 1 e 2; sendo porém menos intensa nas fibras de tipo I, as quais também apresentaram formas hipertróficals. Atrofial de pequenos e grandes grupos de lïbras de ambos os tipos 1 e 2 é observada em músculos severamente afetados pela doença do neurônio motor, sendo patognomônica de desenervação 7 . Os achados musculares caracterizaram uma atrofia neurogênica das fïbras associada com lesões metabólicals. Tais alterações podem ser classificadals dentro de um padrão misto de neuropatia com miopatia. Neste, observa-se a presença de fïbras angulosals e atrólicas, hipertrofia de fibras (primariamente do tipo 1), algumas libras sofrendo fagocitose e fibras com áreas citoplasmáticas de baixà reatividade metabólica (denominadas "mini-cores" e "moth-eaten") !

Os achados histopatológicos medulares do presente trabaltho diferem daqueles observados por Cummings al al. (1990), em relação à marcante presença de neurônios motores tumefatos e cromalíticos, bem como à menor presença de células com corpúsculos citoplasmáticos eosinolílicos ?. A gliose variou conforme a evolução do processo, sendo muito discreta nos calsos crônicos, nos quais predominaram figuras de cicalrizes gliais. $O$ quadro de desmielinização justifica-se pela degeneração walleriana dos axônios, consequiência da morte do soma neuronal ". Apesar das lesões do sistema nervoso central não apresentarem padrão histopatológico idêntico àquele descrito por Cummings el al. (1990), indicam tratar-se da doença do neurônio motor dos eqüinos, uma vez que clinicamente o processo é idêntico ao proposto por estes autores, e as alterações microscópi-

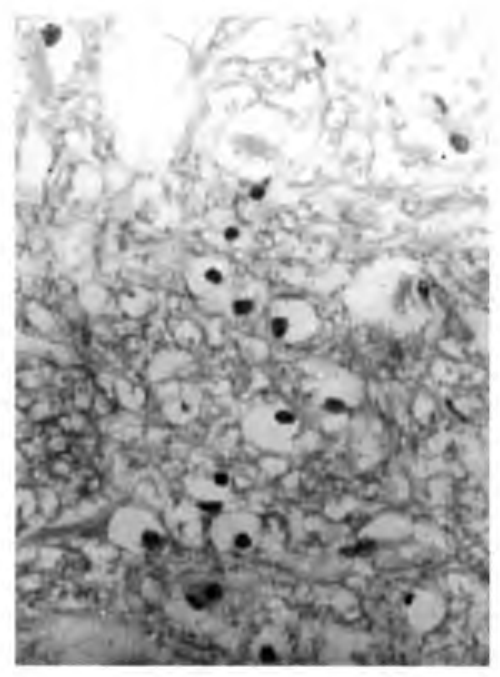

Figura 4

Corno ventral da medula espinhal. Cicatriz glial. HE.2500x. 


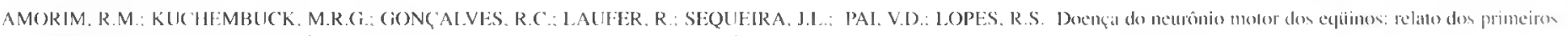

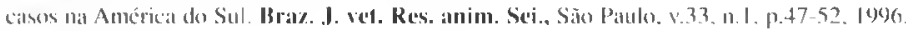

cas demonstraram diminuição acentuada dos neurônios molores inferiores. Tal variação do padrão histopatológico pode estar relacionada com a severidade do processo ou mesmo com diferentes formas de uma mesma doença, a exemplo do yue é observado na esclerose lateral amiotrótica em humanos.

Estudos com eqüinos portadores do processo devem ser realizados com o objelivo de se conseguir avanços na elucidação da eliopatogenia desta neuronopatia, assim como avaliar a possibilidade de a doença do neurônio motor dos equïnos ser utilizada como modelo biológico para estudo da esclerose lateral amiotrófica esporádica.

\section{A ( F A I)ECIMENTOS}

Agradecemos ao corpo veterinário do Regimento de Cavalaria 9 de Julho da Polícia Miliar de São Paulo, pela colaboração e doação dos animais e ao Prol. Dr. H.O. Mohammed, do Departamento de Ciências Clínicass da Universidade Cornell, Nova York, por vir pessoalmente ao Brasil e pelass sugestões propostas.

\section{SUMMARY}

The present paper aims to report cases of equine motor neuron disease in different breeds and ages. The main clinical signs were progressive weight loss, muscle atrophy, generalized weakness, muscle fasciculation and trembling, frequent shifting of support hindlimbs, feet under body, excessive recumbency and death. Clinical diagnosis was confirmed by histopathological studies of the spinal cord, which revealed degeneration and loss of neurons in the ventral horn, particularly cervical and lumbar intumescence. Muscle biopsy revealed neuromuscular disorders with denervation atrophy. The progressive muscle atrophy, weakness and motor neuron degeneration are similar to those in amyotrophic lateral sclerosis, or Lou Gehrig's disease, as described in human beings.

UNITERMS: Neurons; Equidae; Muscular atrophy; Denervation.

\section{REFERÊNCIAS BIBLIOGRÁFICAS}

I-ANI)REWS. F.M.: REED. S.M.: JOJINSON. G.C. Muscle biopsy in the horse: Its indications, lechnicues and complicallons. Veterinary Medicine. v.4. p. 357-65, 1993

2- BANKER, B.Q. The pallonlony of molor neuron disorders. In: ENGEL. A.(i.: BANKER. B.Q. Myology. Part II. New York. McCiraw-llill. 1986. p.2(0.31-67

3 - CUMMINGS, J.F: IDE LAIIUNTA, A: GEORGE: C.: FUIIRER. I. VAIENTINE, B.A.: COOPER, B.J.: SUMMERS, B.A.; HUXTABL.E. ('R.: MOLAMMED, H.O. Eyume motor neuron disealse: a preliminary report. Cornell Veterinarian, v.80, p.357-79, 1900.

4. (UMMINGS, J.F DE LAHUNTA A: MOILAMMED, IIO : DIVERS [.J.: VALENTINE, B.A.: SUMMERS, B.A.: (C)(OPER, B.J. Equino motor neuron discase: a new neurologic disorder. Fquine Practice, v. I.3 1.15-8. 1991.

5- DI: I A]IUNTA. A. Veterinary neuroanatomy and clinical neurology 2.ed. Philadelphia, W.B. Sianders, 1983

6 - DIVERS, TJ: MOIHAMMED, HO: (CUMMINGS, JE: DE LAIUVTA A.: VAL.ENTINE, B.A.: SUMMERS, B.A.: COOI'ER, B.J Equine motor neuren disease: a new callse of weakness, trembling and weigh loss. Compendium on Continuing Education of the Practicing Veterinarian. v. 14, p. 1222-6, 1002

7 - I) (BOWITZ, V. Muscle biopsy: al pratical approach. 2.ed. L.ondon. Bailliere/Tindall, 1985, p.221-87: Diseatses of the lower motor neurone.

8 - 1)(IBOWIT\%, V.: BRRO()KE, M.H. Muscle biopsy: a modem approach. I.ond(on, W, B. Salunders, I)84.

9) IIAIIN, C.N., MAYIIIW, I.Ci. Does equine molor nemon disease exisl in lhe Unifed Kingdon? Veterinary Record, v.132, p.133-4, 1993.

10 - IIGGINS R J - VALDEVELDE M - HOLE EJ - JAGAR J.E COOK L.C.; SIL.BFRMAN. M.S. Neurofibrillary accumulation in the Zebra (Equus burchelli). Acta Neuropathologicar. Berlin, v.37, p. 1-5, 1977

11 - JBB. K.V.F.: KENNEDY. P.C.: PALMER. N. Pathology of domestics animals. t.ed.. ('alifornial. Academic P'ecss. 1993. v.1.

12 - KANEK(). J.J. Clinical biochemistry of domestic animals. 4.ed., Calilomia, Academic Press, 1989.

1.3 - L.AWYER. T.; NETSKY. M.G. Amyolrophic lateral selerosis: a clinicoandomic study of 53 calses. Archives Neurology, v.40, p.667-75. 1981.
14 - LUNA. L.G. Manual of histological staining methods of the Armed Forces Institute of Paflology. New York. MeGraw-Ilill. I968

15- MAYHEW. I.G. Large animal neurology: a hatudbook for velerinary clinicians. Philadelphial, Lea \& Fobiger, 1989.

16- MAYIEW L.G.: DE L AHIUNTA A. WHITLOK R.H.: KROOK. I TASKER. J.B. Spinal cord discalse in the horse. Cornell Velerinarian. v.68. p. 1-207, 1978 (Supplement 6)

17 - MAYHEW. I.G.: WIIITL.OCK. R.II.: TASKER. J.B. Eyuine cerebrospinal lluid: reforences vallues of normat horses. American Journal of Veterinary Research, v.38, p.1271-4. 1977.

I8 - MITSUMOTO, I.: HANSON, M.R.; ('HAI), D).A. Amyotrophlic latteral sclerosis: recent advances in pathegenesis and flecapedutic trials. Archives of Neurology, v.45, p. I 80)-2()2, 1988.

19) - MOIIAMMED. II.O.: CUMMIN( DE LAHUNTA, A.: SUMMERS, B.: IAARIROW, B.R.I I.: TREMBICKI (iRAVISS, K.: MAUSKOIT. A. Risk faclors associaled with equine motor neuron discase: a possible model for human MNI). Neurology. V.43, P.966-7I, 1093.

20) - PEARSON. E. (i.: (iRAICi. A.M. The diagnosis of liver discase in equine and food anmimals. II. Modern Veterinary Practice, v.61. p.315-20). 1980 .

2I - PFTER, I.B : BARNARD, R.J.: FDCIERTON, V.R. Metabolic proliles of duree dibers lypes of skelelal muscle iu guincil-pigr and rabbits. Biochemistry, Washington, v.II, 1).2627-33,1972.

22 - 'TANDAN, R.: BRADLEY,W. i. Molor' neuron discase. In: ASBLRY, A.K MCKHANN, G.M.: MCDONALD. W.I. Diseases of the nervous system: clinical neurobiology. Philadelphia, W.B. Silunders, v.2, p. 1239-57, 1986.

23 - VALENTINE, B.A ; DE IAIIUNTA, A.; GEORGE, C.: SUIMMERS, B.A. C'UMMINGS, J.F.; DIVERS, T.J.; MOHAMMMED, II.O. Commenlary: acquired equine motor neuron discatse. Veterinary l'athology, v.31. p.13()-8. 1994

24 - WILLIAMS. D.B.; WINIDEBANK, A.J. Molor neuron disealse (amyotrophlic lateral sclerosis). Mayo ( Clinic Proceedings, v.66, p.54-82, 1901.

Recebido) para publicaçáo: (04/04/94 Aprovado para publicaçao: 19/06/95 